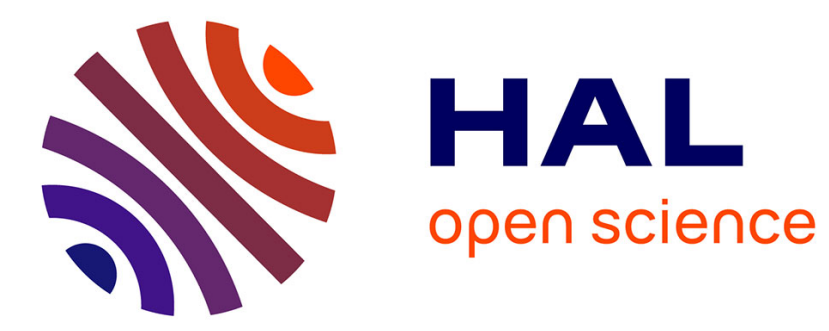

\title{
Multimodal approach to automobile driving comfort: the influence of visual setting on assessments of vibro-acoustic comfort in simulators
}

\author{
Maël Amari, Etienne Parizet, Vincent Roussarie
}

\section{- To cite this version:}

Maël Amari, Etienne Parizet, Vincent Roussarie. Multimodal approach to automobile driving comfort: the influence of visual setting on assessments of vibro-acoustic comfort in simulators. Applied Acoustics, 2013, 74 (12), pp.1378-1387. hal-00842580

\author{
HAL Id: hal-00842580 \\ https://hal.science/hal-00842580
}

Submitted on 29 Jul 2013

HAL is a multi-disciplinary open access archive for the deposit and dissemination of scientific research documents, whether they are published or not. The documents may come from teaching and research institutions in France or abroad, or from public or private research centers.
L'archive ouverte pluridisciplinaire $\mathbf{H A L}$, est destinée au dépôt et à la diffusion de documents scientifiques de niveau recherche, publiés ou non, émanant des établissements d'enseignement et de recherche français ou étrangers, des laboratoires publics ou privés. 


\title{
MULTIMODAL APPROACH TO AUTOMOBILE DRIVING COMFORT: THE INFLUENCE OF VISUAL SETTING ON ASSESSMENTS OF VIBRO-ACOUSTIC COMFORT IN SIMULATORS.
}

\author{
AMARI Maël ${ }^{\mathrm{a}, 1}$, PARIZET Etienne ${ }^{\mathrm{b}}$, ROUSSARIE Vincent ${ }^{\mathrm{a}}$ \\ (a) PSA Peugeot Citroën, route de Gisy, F-78943 Vélizy-Villacoublay Cedex \\ (b) LVA INSA-Lyon, 25 bis av. Jean Capelle, F-69621 Villeurbanne Cedex, \\ etienne.parizet@insa-lyon.fr (corresponding author)
}

\begin{abstract}
The growing use of vehicles in urban areas means that comfort in low-speed driving phase is an increasingly important consideration for manufacturers. Examining comfort in such situation requires not only field experiments but also the use of simulators to control the characteristics of the stimuli to which participants are exposed.

Incorporating a visual scene with vibration and sound stimuli is one possible way of improving simulation conditions. Two experiments were conducted to measure the influence of the immersion relating to vibratory, sound and visual modes on comfort assessments. In the first experiment, participants were exposed to different combinations of vibration, noise and road videos ( 8 vehicles, 2 roads). The visual mode only led to a small bearing on ratings. Regardless of the mode presented, the level of vibration emerged as the crucial factor in assessing overall comfort. Little interactions between sound and vibration modes were also highlighted. The second experiment dealt with context given by the visual mode. Acoustic and vibration stimuli were combined with very different road videos, which modified participants' expectations with regard to the situation. Results demonstrated that combining different visual settings with vibro-acoustic stimuli, no matter how much they were opposed, only exerted a small influence on participants' comfort.

In the end, with regard both to immersion and contextualisation, the effects of the visual mode are so low-scale as to be insignificant. Accordingly, they may be ignored in future similar experiments, which can make the experimental set-up easier.
\end{abstract}

Keywords : sound and vibration perception

\section{INTRODUCTION}

The growing use of vehicles in urban areas means that comfort in low-speed driving phase is an increasingly important consideration for manufacturers. In this situation vehicle comfort is tied to vibro-acoustic comfort. The main sources of constraints in the occupant's environment are vibrations and sounds transmitted to the cabin (vibrations from the floor pan, pedals, seats, steering wheels and dashboard and engine and road noise).

\footnotetext{
${ }^{1}$ Present address : Institut National de Recherche et de Sécurité, rue du Morvan, CS 60027, F-54519 Vandoeuvre les Nancy, mael.amari@inrs.fr
} 
Examining comfort in low-speed driving phase requires not only "in situ" experiments but also the use of simulators. Unlike in the real-life situation, in simulations it is possible to control the characteristics of the stimuli to which participants are exposed. Sound and vibration are the basicmodes of this immersion. However, constraints relating to operating costs prevent the identical reproduction of an automobile cabin.

Incorporating a visual scene with vibration and sound stimuli is one possible way of improving simulation conditions. Projecting videos on to a screen is a low-cost method of making the laboratory environment more realistic. While it is legitimate to assume that visual mode cannot directly be involved in assessments of vibro-acoustic comfort, it does provide contextual information on situations that are apt to influence assessments. Therefore, it is worth exploring the role of the information provided by the visual mode: in this precise case, can videos alter participants' feelings or are they merely used to make the situation appear more realistic?

Most of the studies available on automobile driving comfort consist of individual research into each of the vibration, sound and visual modes. This type of study does not factor in people's capacity to integrate stimuli from another mode. Low-speed driving comfort should be studied in conditions as close as possible to those prevailing in an automobile cabin.

Genuit et al. compared the assessment of vehicle noises carried out "in situ", in a simulator (sound + vibrations or just vibrations) and in a listening laboratory (just sounds) [1]. The results showed a strong correlation between experiments. Differences in the use of the rating scale emerged, with participants using a lower rating dynamic in the simulator than in vehicles. In addition, results were very similar to those obtained when participants only evaluated vibrations in a simulator. The rating scale was also reduced when sounds were assessed by themselves. The author underlined the fact that contextual differences (driving task, absence of inertia force, visual scene, etc.) constituted a possible explanation for the dispersion of results. In spite of these observations, assessments in simulators remain close to those performed in the field.

Other studies have also shown that vision could exert a strong influence on subjective assessments of sounds. Viollon et al. undertook a laboratory experiment to assess how judgment of an urban environment sound may be affected by the simultaneous presentation of visual scenes representing different level of urbanisation [2]. Generally speaking, it seems from the two scales of assessment used (of a "pleasant" and "stressful" nature) that the more an environment is urban, the less noise is welcome. This effect is not identical for all tested sounds and depends on the semantic context of the noise - it is pronounced for natural sounds and absent for man-made sounds. In the automobile field, Ellermeier et al. evaluated the influence of visual contextualisation on the assessment of sounds in a laboratory [3]. Participants assessed the power of sound sequences combined with images of more or less powerful vehicles. It was found that the image of a powerful car induced a positive bias in assessments. The reverse was true for low-power cars. This suggests that participants incorporate the visual mode into their assessments even when explicitly asked to assess only the sound mode.

Other studies also shown that even a basic sound attribute such as loudness can be modified by visual stimuli. Menzel. et al. related that the color of a sports car influence the loudness evaluation of the accelerating sound [4]. A similar effect could be found in the case of highspeed train outside noise ([5], [6]), though high inter-individual differences may reduce this assertion [7]. 
If most documented effects concern visual and sound modes, some interaction can occur between vibration and other modes too [8]. That is why when it comes to automobile driving comfort, we are entitled to expect that seeing a road fault beforehand can alter the degree of disturbance that a participant feels it when the vehicle drives over it.

Two experiments were conducted to measure the influence of the simulated environment on comfort assessments:

- In the first experiment, participants were exposed to different combinations of vibration, noise and road videos during assessments of vibro-acoustic comfort.

- The second experience dealt with context given by the visual mode. Acoustic and vibration stimuli were combined with very different road videos, which modified participants' expectations with regard to the situation.

\section{EXPERIMENT APPARATUS}

To assess vibro-acoustic driving comfort in a simulator, the vibrations and sounds measured in vehicles' cabins must be acurately reproduced. This reproduction must be accompanied by the projections of videos matching the passenger's field of vision.

\subsection{Measurements}

The objective is to measure vertical vibrations in the passenger seat, the noise environment in the vehicle interior and the passenger's field of vision on different roads and for several vehicles. To this end, an accelerometer (Type ICP - PCB) is placed vertically on the front screw of the right slider of the seat. The pressure field at passenger's head level is recorded with a dummy head (HMS III - Head Acoustics) fitted on to the seat. The video footage of the road is recorded with a HD digital camera (HDR HC1E - Sony) fitted on to the windscreen. The field leaves out the dashboard so as to avoid including any components that could give away the identity of the vehicles in the videos.

The accelerometer and acoustic head are linked to a front end (Octobox - Head Acoustics). The acoustic and vibrations measurements are saved into a computer (Head Recorder \& Artemis 8 - Head Acoustics). Sound and vibrations are measured at sampling frequencies of $48 \mathrm{kHz}$ and $1.2 \mathrm{kHz}$ respectively with 24-bits quantification. Video resolution is $1920 \mathrm{x} 1080$ pixels. Synchronisation between the front end and the camera is achieved using a clocking signal connected to the two systems.

8 vehicles, each a different brand, were selected for the measurement of noise, vibration and visual stimuli. All of the vehicles were mid-range ( $\mathrm{M}$ segment) and had 4-cyclinder engines (5 diesel engines and 3 petrol engines).

Samples of four sections of different roads were chosen for recordings that lasted six seconds. Vehicles cruised in $2^{\text {nd }}$ gear at $30 \mathrm{kph}$. The surface of the chosen road sections were more or less deteriorated and appearances varied substantially (Figure 1):

- Road A was a country road with rough surfacing. It had an even appearance and the extracted segment reflected a steady driving situation; 
- The Road B section included a change of surface. The vehicle drove from a part of the road that was in good condition to an older, much more degraded stretch. The switch to the deteriorated part amounted to a very clear vibro-acoustic event;

- Road C featured new surfacing with no irregularities. It had a very smooth appearance and matched a very comfortable and regular situation;

- Lastly, Road D was severely deteriorated and featured many irregularities. The scale of the distortion of the causeway caused vertical displacements of the visual scene, which clearly suggested a highly uncomfortable situation.
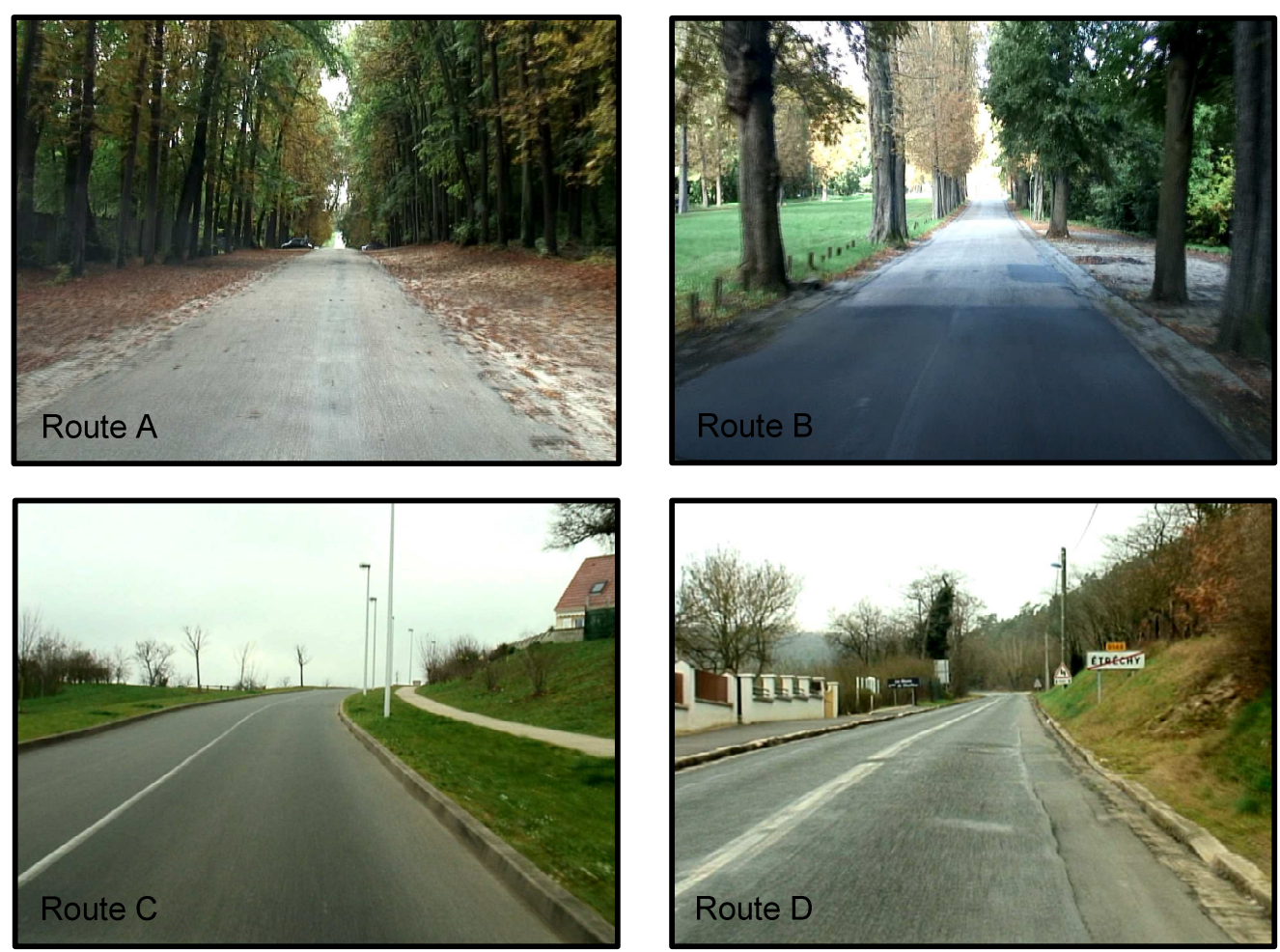

Figure 1: video excerpts of the 4 roads

These reflected a varied choice of situation. The aim was to instil different expectation into the participants in terms of vehicle comfort. The steady course of Road A guided assessment towards the vehicle's capacity to filter sounds and the irregularities of the surface, whereas Road B confronted the vehicle and the passenger with a more pronounced obstacle. The cause of the vibration and sound effects was thus perceived as "external" to the vehicle. The second two situations conveyed the most extreme cases. Road C seemed perfectly comfortable, while Road D appeared to be very uncomfortable.

\subsection{VIBRATION TEST BENCH}

A vibration test bench featuring a platform on four springs and fitted with a vehicle seat was used to conduct the experiments (Figure 2). A detailed description of this device is given in [9]. Please note that, if it is now possible to reproduce vertical and longitudinal vibrations, as it can be seen in figure 2, the vertical vibration stimulation only was possible at the time this experiment was conducted. The platform was vertically activated by a shaker (V555E - LDS) 
steered by an amplifier (PA1000 - LDS). The device was used at a frequency band of 2-100 $\mathrm{Hz}$, due to the limited displacement of the shaker $(25.4 \mathrm{~mm}$ pk-pk). This is a limitation of the bench, as measurements exhibit frequencies lower than $2 \mathrm{~Hz}$. Recordings were filtered to compensate for the effects of the mechanical transfer of the system.

Road videos were projected onto a $2 \times 3 \mathrm{~m}$ screen positioned $3 \mathrm{~m}$ from the participants. The vibration test bench included a vehicle seat and enabled replication of the vibrations recorded in vehicles. Laboratory background noise was $35 \mathrm{~dB}(\mathrm{~A})$ at the participant's head position. Reverberation time was $0.1 \mathrm{~s}$. The only source of sound within the laboratory was the ventilation of the shaker. This sound was independent of the vibration signal and completely hidden during tests.

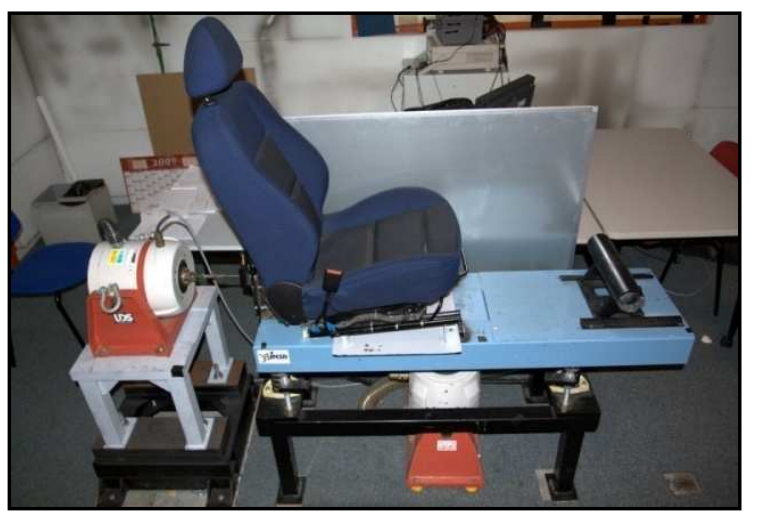

Figure 2: Vibrating test bench

The sound system consisted either of electrostatic headphones (HA II \& PEQ HPS IV - Head Acoustics) or loudspeakers (7050A - GENELEC). Both were supported in the low frequenciy range (below $100 \mathrm{~Hz}$ ) by a subwoofer (8030A - GENELEC). All of the apparatus was positioned in front the participant, behind the screen and hidden by curtains. When loudspeaker enclosures and subwoofers being used, binaural recordings of the acoustic head were transauralized with a special software (Génétrans - GENESIS). This step established correct spacing of binaural recordings. Lastly, when headphone were used, signals were filtered to compensate the effect of the conchae [10].

The whole apparatus was controled by a computer with built-in sound card $(1820 \mathrm{~m}$ Creative) and a video projector (VPL CX70 - Sony). Participants were put into a situation representing the vibro-acoustic environment of a car in low-speed driving phase. It is important to point out that the vibrations recorded were correctly reproduced at the slider and not at the interface between the seat and the participant. Reproduction did not, therefore, correspond to real vehicles, since the effect of the seat was not taken into account.

The physical characteristics of the stimuli in the simulator were measured. A variety of indicators conventionally found in literature were considered for the sounds and vibrations. The equivalent continuous A-weighted sound pressure level (LAeq) and loudness (calculated according to the ISO-532B standard [11]) were calculated for each sound. For vibrations, Vibration Dose Value and the rms weigthed acceleration (Lv) were computed according to ISO-2631 standard ([12], [13]). Lastly, Table 1 shows the vibrations and sound levels of stimuli. 


\begin{tabular}{|c|c|c|c|c|c|c|c|c|c|c|}
\hline & $\begin{array}{c}L_{-} \text {Vib } \\
(\mathrm{dB})\end{array}$ & $\begin{array}{l}\text { VDV } \\
\left(\mathrm{m} / \mathrm{s}^{1.75}\right)\end{array}$ & $\begin{array}{l}\mathrm{SPL} \\
(\mathrm{dB})\end{array}$ & $\begin{array}{l}\mathrm{SPL} \\
(\mathrm{dBA})\end{array}$ & $\begin{array}{c}\mathrm{N} \\
\text { (sone GF) }\end{array}$ & $\begin{array}{c}\text { L_Vib } \\
(\mathrm{dB})\end{array}$ & $\begin{array}{l}\text { VDV } \\
\left(\mathrm{m} / \mathrm{s}^{1.75}\right)\end{array}$ & $\begin{array}{l}\mathrm{SPL} \\
(\mathrm{dB})\end{array}$ & $\begin{array}{l}\mathrm{SPL} \\
(\mathrm{dBA})\end{array}$ & $\stackrel{\mathrm{N}}{\text { (sone GF) }}$ \\
\hline V1 & 102.3 & 0.28 & 85.3 & 49.7 & 6 & 103.7 & 0.34 & 87.1 & 56.6 & 9.4 \\
\hline V2 & 121 & 2.80 & 82.3 & 52.0 & 6.8 & 116.7 & 1.50 & 80.8 & 49.6 & 5.7 \\
\hline V3 & 116.2 & 1.41 & 78.7 & 49.8 & 5.5 & 113.1 & 0.99 & 76.4 & 48.6 & 5.1 \\
\hline V4 & 105.3 & 0.39 & 85.3 & 49.5 & 5.9 & 104.9 & 0.37 & 84.7 & 48.4 & 5.5 \\
\hline V5 & 103.7 & 0.32 & 78.9 & 50.3 & 5.7 & 102.6 & 0.28 & 79.3 & 49.4 & 5.3 \\
\hline V6 & 104.2 & 0.35 & 78.5 & 49.3 & 5.5 & 101.7 & 0.26 & 76.4 & 46.9 & 4.6 \\
\hline V7 & 104.3 & 0.35 & 86.3 & 48.3 & 5.5 & 102.6 & 0.29 & 83.3 & 47.3 & 5 \\
\hline V8 & 117.4 & 1.58 & 78.0 & 55.8 & 5.5 & 114.1 & 1.08 & 74.5 & 46.1 & 4.5 \\
\hline
\end{tabular}

Table 1: Characteristics of stimuli. Left part of the table : road A. Right part : road B.

\section{InFLUENCE OF VIBRATION, SOUND AND VISUAL SETTINGS (EXP. 1)}

In this experiment participants were exposed to different combinations of sound, vibrations and road videos.

\subsection{PROTOCOL}

The stimuli chosen for this experiment were the sounds, vibrations and videos of eight vehicles, recorded on Roads A and B. These two roads were selected because videos show many visual informations : the rough surfacing of road A can be clearly seen as well as the sudden surface change of road B. The goal of this experiment was to evaluate the influence of this visual information on the evaluation of comfort in the car.

The chosen sound reproduction system consisted of loudspeakers (and the subwoofer) as this created a situation close to an in vehicle one for the participants.

64 volunteer participants ( 47 men, 17 women) were recruited to take part in the experiment. All were in good health. No other particular conditions were required. The participants took their place on the bench and were invited to adopt a comfortable sitting position. They had to imagine themselves sitting in the cabin of a real car with the windows closed and air conditioning turned off.

Three modal patterns were presented to the participants in three types of tests: VIS, VS and V. The VIS tests included vibrations, images and sounds. The VS tests comprised vibration and sounds, while the $\mathrm{V}$ tests only used vibrations. For each of the two roads, the video of a single vehicle (V1) was used for all vehicles. All distinctive features that could have enabled a participant to identify the vehicle during evaluations were removed. This step did not alter the synchronisation of the videos or the acoustic and vibration stimuli. 
For each type of test, participants were presented with samples measured from the eight vehicles on the two roads. This meant that, for each road, participants undertook three tests VIS, VS and V.

The participants took part in six tests in a different order, one after another. The order in which the tests were presented was not entirely balanced. The presentation order of the two roads and the VS and V tests were balanced, however only half of participants began with a VIS test, whereas the other half finished with this test. The assumption here was that only the visual mode influences participants' awareness of the context of the situation, therefore only participants who have undergone a VIS test before a VS or V test know exactly what situation the vehicle was in. By not ensuring total balance of the presentation order of the VIS tests we struck a methodological compromise between the evaluation of the effect of the knowledge of the visual context on the other modes and the number of participants in the experiment. This minimised the number of participants in each of the two categories.

Furthermore, within each test, the vehicles presentation plan was such that each participant experienced a different series. Each test began with three examples and all stimuli were repeated twice. The participants assessed all of the vehicles before undergoing the repetitions. Each stimulus could be played as many times as necessary.

Participants had to assess the overall comfort of the situation. They gave their answers by placing the cursor on a continuous graduated scale featuring the following labels, which were assigned numerical values:

- "Not at all comfortable" (0),

- "Not really comfortable" (25),

- "Relatively comfortable" (50),

- "Comfortable" (75),

- "Very comfortable" (100).

This five levels scale was selected according to the recommendations of Fields et al. [14].

\subsection{Results}

The data to be processed was spread across six separate tests, corresponding to the three combinations of modes -VIS, VS and V - examined on Roads A and B. For each of these tests 64 people performed two assessments of the recordings of eight vehicles. This spread of results shaped how the data was processed. After evaluating the degree of consensus among participants and the level of discrimination according to vehicles for each test, the effects of the various experiment settings were ascertained. They were illustrated by descriptive statistics and the links between observations and the physical properties of signals were highlighted.

First of all, the consensus between participants was evaluated. An Ascending Hierarchical Classification (AHC) was produced for the data from each test (Ward Method). Results were comparable for each of the six tests - no participant category emerged. These observations were confirmed by internal mapping (Principal Component Analysis: vehicles as individuals/participants as variables). All participants were grouped on the first axis (defined by the first eigenvector of the data covariance matrix), which always represented more than $80 \%$ of total inertia. None of the other axis exceeded $6 \%$ of inertia. It can be seen, then, that these initial analyses found a very high degree of consensus among participants. 
The degree of discrimination between vehicles was also estimated using average comparison tests (tests HSD - $\mathrm{p}<0.05$ ). Through these average comparison tests it was possible to split products into groups on the basis of statistical comparison performed in pairs. These demonstrated a very high degree of discrimination (between five and eight groups across all tests).

The order in which tests were presented was conceived so that half the participants were shown a visual setting first and the other half saw it last. By comparing the data of these two groups we could estimate the effect of the knowledge of the visual setting on the vibroacoustic assessments.

As before, the levels of consensus and discrimination were assessed. In both cases the appropriate analysis found that awareness of the visual context did not exert any effect on the assessments of comfort.

Figure 3 shows the averages obtained by vehicles for Roads A and B when VIS tests were presented first and when they were not presented first. The results are shown with their confidence intervals of $95 \%$. The VIS curves were deliberately left out of this graph to make it easier to read. It appears that participants who were submitted to the VIS condition at first gave evaluations slightly higher than participants who were submitted to the whole set of stimuli at the end. This is true for most cars, which indicates that the knowledge of the visual aspect of the road may make participants more indulgent in terms of their assessment of incabin comfort. Nevertheless, these differences are small as regard to differences between cars. Therefore, the 64 participants were grouped together for the remainder of the analysis.

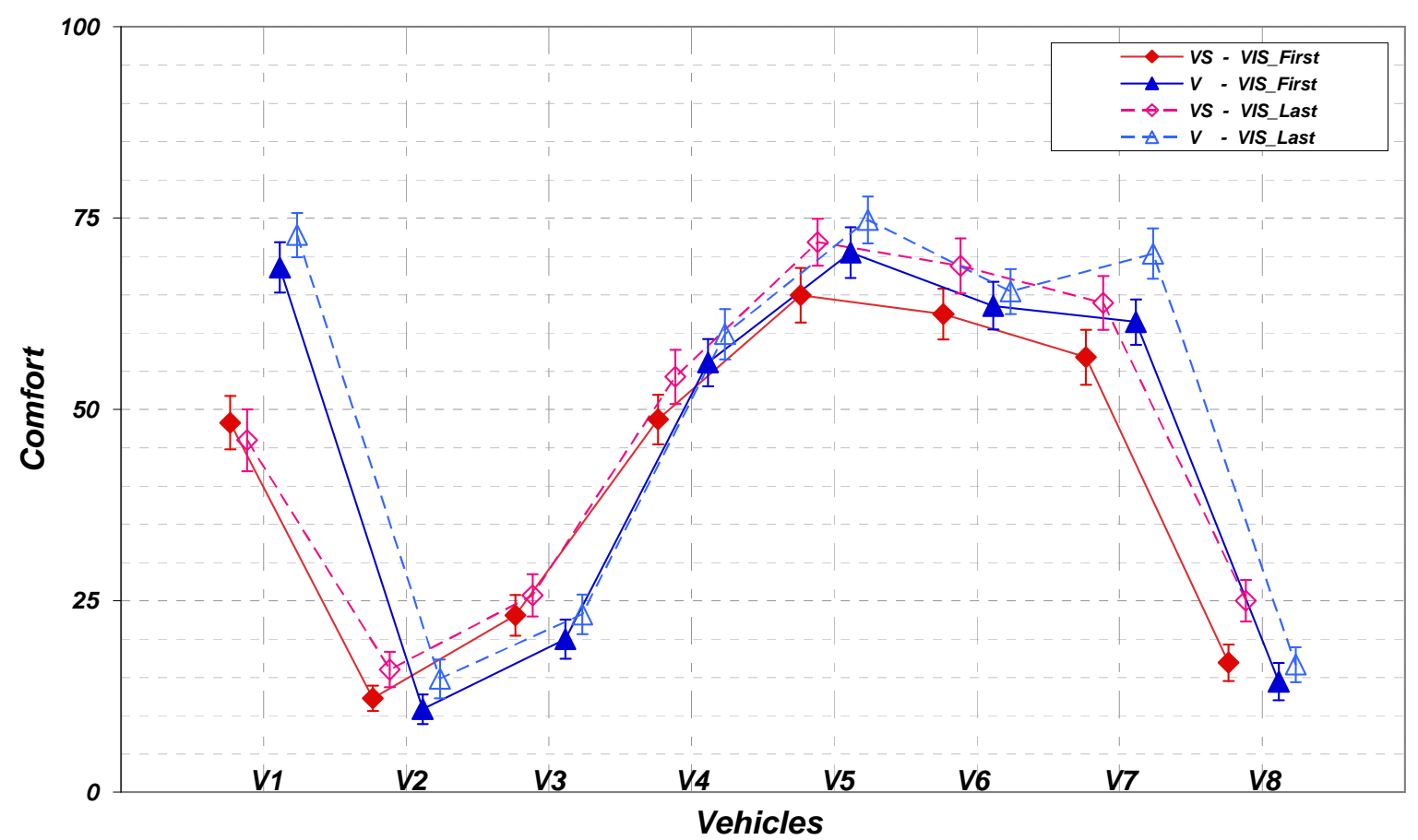

Figure 3: Effect of awareness of the visual context

We then estimated the average ratings (over the three tests) of vehicles on Roads A and B (Figure 4). Comfort ratings were similar for both roads. An ACH revealed two groups of vehicles. Five vehicles (V1, V4, V5, V6 and V7) were rated "Quite Comfortable" (50) and 
"Comfortable" (75) while three others (V2, V3 and V8) were rated "Not Really Comfortable" (25). Ratings for Road B grew and ratings differed significantly from one road to another for all vehicles except V4. The test results for Road A and B were processed differently for the rest of the experiment.

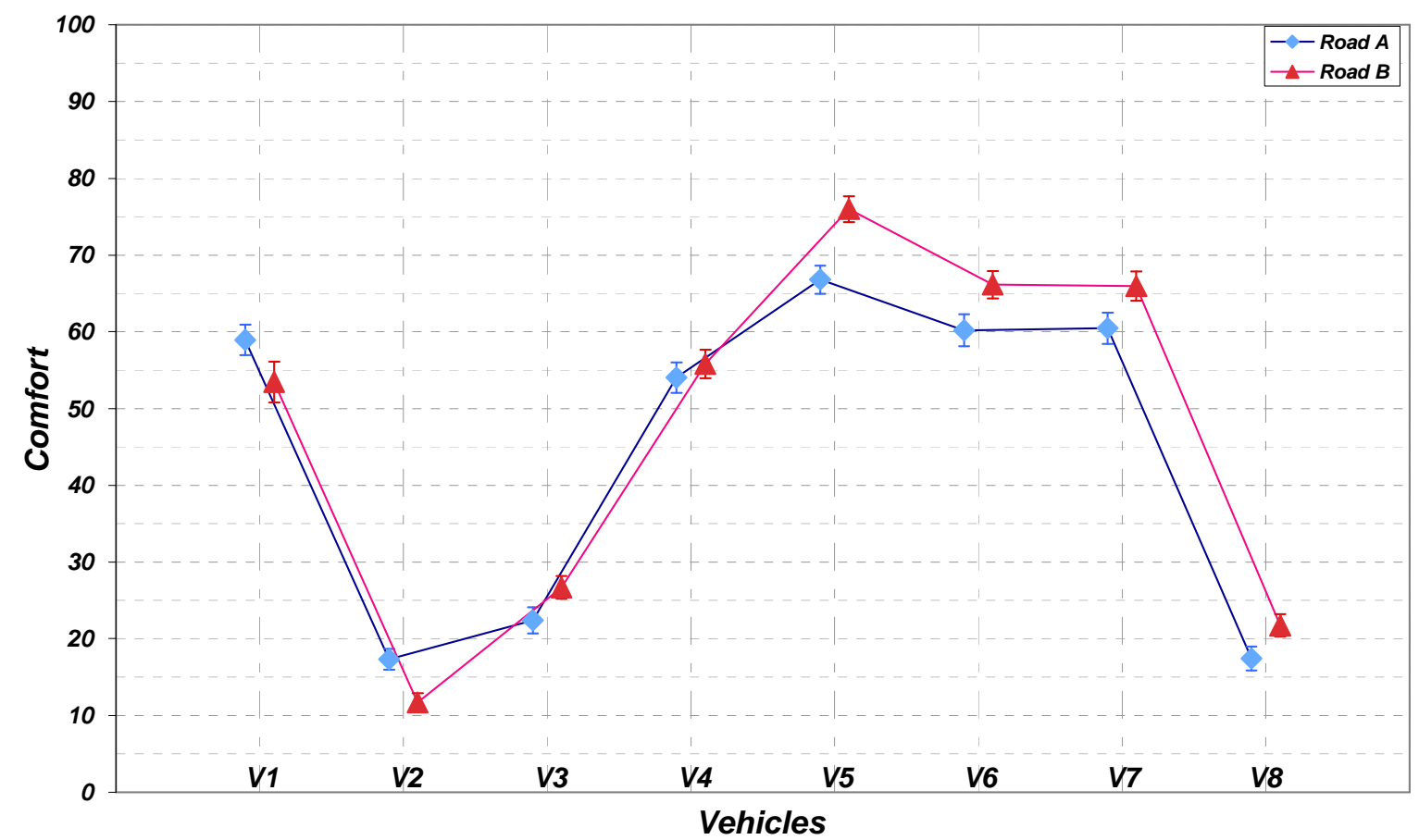

Figure 4: Effect of road type

Before establishing the effect of the different modalities exactly, an overview of each of the parameters of the stimuli presentation was carried out. Analyses of variance were made of the data for each of the tests. These ANOVAs consisted of a comparison between the statistical gaps for each of the 64 participants, eight vehicles and two repetitions. The dependent variable was the comfort rating. All the main effects of the factors and their first order interactions were included in the model. The participant factor was set as random in the model. The results of the ANOVA were, therefore, independent of the sample of participants who has sat the tests.

The ANOVA results were identical for the six tests. The "vehicle" effect was systematically significant while the "Repetition" effect was not. The F values clearly showed that the vehicle factor was responsible for the vast majority of result dispersion. It is important to note that the influence of other parameters is secondary to the difference across vehicles. Lastly, additional analyses of the data from each test confirmed that the "repetition" effect is not significant. These are considered as supplementary assessments of the vehicles.

\subsubsection{EFFECTS OF MODES}

The effect of the modes on the consensus, vehicle discrimination and comfort assessment were then evaluated.

Firstly, the six internal data maps for each test were compared. From this it became clear that the inertia explained by the first axis as determined by a Principal Component Analysys decreased by $92 \%$ on average on the two roads for the V tests, $82 \%$ for the VS tests and $81 \%$ 
for the VIS tests. This slight reduction may reflect a certain variability between individuals in the way they processed sound and vibrations modes. Sound causes an increase in the dispersion of participants. The modal setting had no significant influence. It should be specified that these results showed a clear consensus and that this dispersion is due to a minority of participants.

With regard to discrimination, average comparison tests (HSD - $\mathrm{p}<0.05)$ found that, for each of the roads, vehicles were split into five distinct groups for V tests, seven for VS tests and eight for VIS tests. Increases in discrimination were observed for vehicles deemed "Not Really Comfortable" (V2 and V8) and those judged "Comfortable" (V1, V2 and V8). The increased discrimination observed in VS tests may be explained by the differences in sounds in the vehicles, which made it easier to distinguish between them. However, this argument cannot be applied to the visual mode because the same road videos were projected for all vehicles.

Figures 5 and 6 show the comfort ratings for each mode for the eight vehicles on Roads A and B. The effect of the modes was slight compared to that of the vehicles. The three curves are quite similar, which suggests that participants were able to assess the comfort of a situation when they were only exposed to a vibration mode, whose role, therefore, appears to be dominant in the assessment of comfort. Comparison of the VS and V curves shows that the effect of sounds fluctuates. While no general trend was observed, it was noticeable that sound has a very significant influence on assessments of V1, whose score went from an average of 70 for V tests to 47 for VS tests and 51 for VIS tests. Lastly, it could be observed that for the two roads and for vehicles (except V6 on Road A) the VIS curve was slightly higher than the VS curve. The average gap across all vehicles was +5 points. Application of the visual mode pushed ratings closer to "Comfortable". This mode was not, however, as important as sound mode. Finally, it should be noted that nothing - not the stimuli and not the protocol - explains the variance observed between the VIS tests and the other for V6 on Road A.

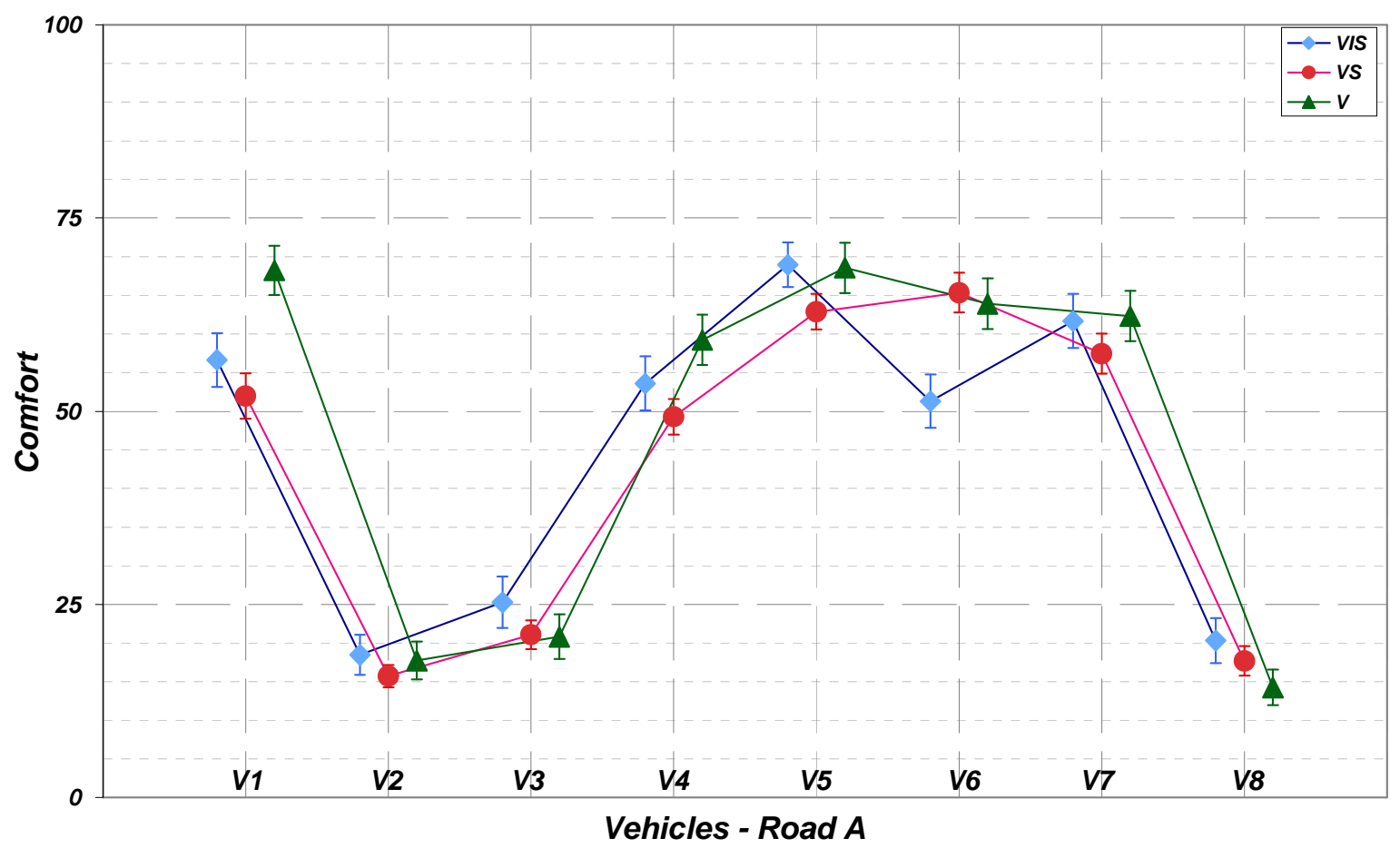

Figure 5: Effect of modes - Route A 


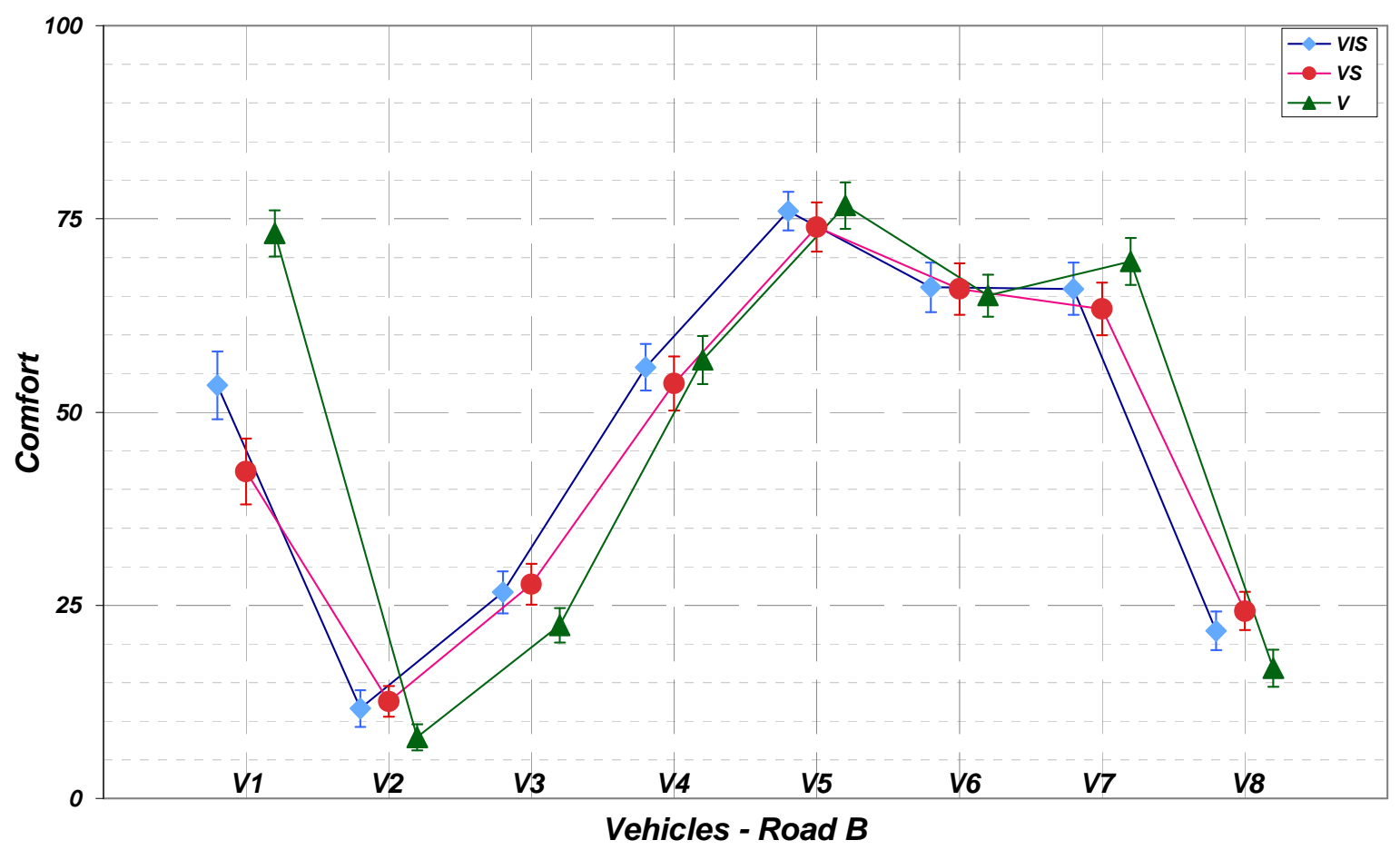

Figure 6: Effect of modes - Route B

Average comparison tests (tests HSD - p < 0.05) carried out on each of the mode configurations showed that the differences between the V and VS tests were significant for three vehicles on Road A and for five on Road B. The proportions were almost identical for $\mathrm{V}$ and VIS tests ( 3 vehicles for Road A and 4 for Road B), even though the same vehicles were not involved. As for the differences between VIS and VS, three vehicles were different for Road A and just one for Road B. This imbalance arising from the visual mode attests to the different influences of the two road types.

In conclusion, the presence or absence of visual or acoustic modes altered assessments of comfort. The influence of the two modes was not the same. The influence of sound was found to be significant for some vehicles but no trend could be discerned. The visual mode led to greater discrimination between vehicles and was reflected slightly in the ratings.

\subsubsection{Signal PROPERTIES}

The various assessments were approximated by combinations of the physical parameters represented in table 1.

When participants were submitted to vibrations only, assessments were highly correlated with the vibration rms values expressed in $\mathrm{dB}\left(\mathrm{R}^{2}=0.93\right)$. Nevertheless, one has to be cautious about this correlation as there clearly exist two groups of cars : levels in V2, V3 and V8 are between 113 and $120 \mathrm{~dB}$, while levels in the other cars are between 102 and $106 \mathrm{~dB}$.

When participants were submitted to sound and vibration stimuli, it appeared that assessements could be represented by a linear combination of vibration rms values and Aweighted sound level :

$$
\text { Score }=413-1.06 \mathrm{LA}_{\mathrm{eq}}-2.92 \mathrm{~V}_{\mathrm{rms}}
$$

This model is significant $\left(\mathrm{R}^{2}=0.9, \mathrm{~F}(2,13)=56.3, \mathrm{p}<0.001\right)$. 
This model explains the decrease of comfort of vehicle 1 between conditions V and VS on road $\mathrm{B}$. This is due to the noise level in that car, which is significantly higher than level in the other vehicles.

\section{VISUAL SCENE AND SOUND SYSTEM (EXP. 2)}

The acoustic and vibration recordings accompanying the various visual contexts changed participants' expectations from situations. In order to get more pronounced displays of the anticipated effects, a wide variety of arrangements were chosen for the visual scenarios.

The sound reproduction system was also altered : the transauralized set-up was replaced by headphones (plus a subwoofer for improving the low-frequency range). For many applicative studies, headphones are recommended for playing back dummy-head recordings, as they allow more faithful reproduction of the in-cabin sound field. On the other hand, using headphones placed the participant into a situation that is farther removed from reality. A secondary goal of this experiment was to evaluate whether the influence of videos can be different when using headphones instead of loudspeakers for sound reproduction.

\subsection{PROTOCOL}

60 participants (40 men, 20 women), of whom 20 had taken part in the first experiment, were hired on a volunteer basis. Sitting guidelines and instructions were the same as for the previous experiment.

The vibration, sound and visual stimuli were presented to participants simultaneously. Acoustic and sound signals were selected according to the eight vehicles (V1-V8) recorded on Road A (deteriorated road). These signals were presented along with video recordings of Road A, Road C (smooth road) and Road D (heavily deteriorated road). Road B was not used because it featured a very striking switch from a smooth surface to a rough surface, which was not reflected in the acoustic and sound signals. The total number of stimuli was 24 .

Participants were exposed to identical vibro-acoustic stimuli, accompanied by three very different situation scenarios. |We worked on the assumption that in the presence of Visual C, the vibro-acoustic environment was amplified. By contrast, the vibro-acoustic stimuli relating to Visual D were considered mitigated. The objective was to put participants in artificially exaggerated situations.

Stimuli were assigned to three tests according to visual scene (A, C or D) and were presented in turn to the participants. The presentation order of the tests was balanced so as to overcome any bias relating to this parameter. All of the tests began with the presentation of three examples. For each test, the signals of the eight vehicles were repeated twice. Participants assessed all of the vehicles the first time before undergoing the repetitions. Participants were allowed to replay the stimuli as often as they wished.

Participants had to gauge the overall comfort of each situation. The rating scale was identical to the one used in the previous experiment (continuous scale with five grades, ranging from "Not at All Comfortable" to "Very Comfortable"). Instructions were presented along with a verbal description of the situation. Subject did not know the real objective of the experiment. There was a short break between each test. The total duration of the experiment was 30 minutes. 


\subsection{Results}

The chosen protocol was such that the data to be processed was spread within independent tests, corresponding to Visual A, C and D and vibro-acoustic recordings for Road A. In each of the tests 60 people assessed two reproductions of the recordings of the 8 vehicles.

The first analyses were aimed at evaluating the consensus among participants and their level of discrimination of vehicles. Then the effect of the visual context on the consensus, vehicle discrimination and evaluation were measured.

The first step was to perform a clustering of the data from each test. The tree diagrams obtained through this are used to distinguish the categories of participants whose breakdown seems imbalanced. Two categories of participants for Tests A $(20,40)$ and C (33, 27) emerged in this way, while three emerged for Tests D (27, 24 and 9). Lastly, since only a small number of participants were in the third category for Tests D, a compromise was reached as participants for all tests were segmented into two groups using the K-mean method $(34,26)$.

By comparing raw data to centred data and then to centred and reduced data, it was possible to relate participant categories through different uses of the rating scale. Participants in the first category gave higher ratings to the most comfortable vehicles.

Follow-up analyses were carried out to determine the source of this segmentation. First, a Khi 2 test demonstrated that this participant segmentation had nothing to do with whether or not the participant took part in the first experiment. Comparing the results of Test A to the VIS test (Road A) from the previous experience established the influence of the sound reproduction method on comfort assessments. Figure 7 presents the average ratings obtained by vehicles in each of the two experiments. From the comparison of the two participant categories of Experiment 2 it is clear that the first category is close to the results obtained with loudspeakers. Therefore, using headphones has the effect of reducing the rating dynamic for half of the participants (Category 2).

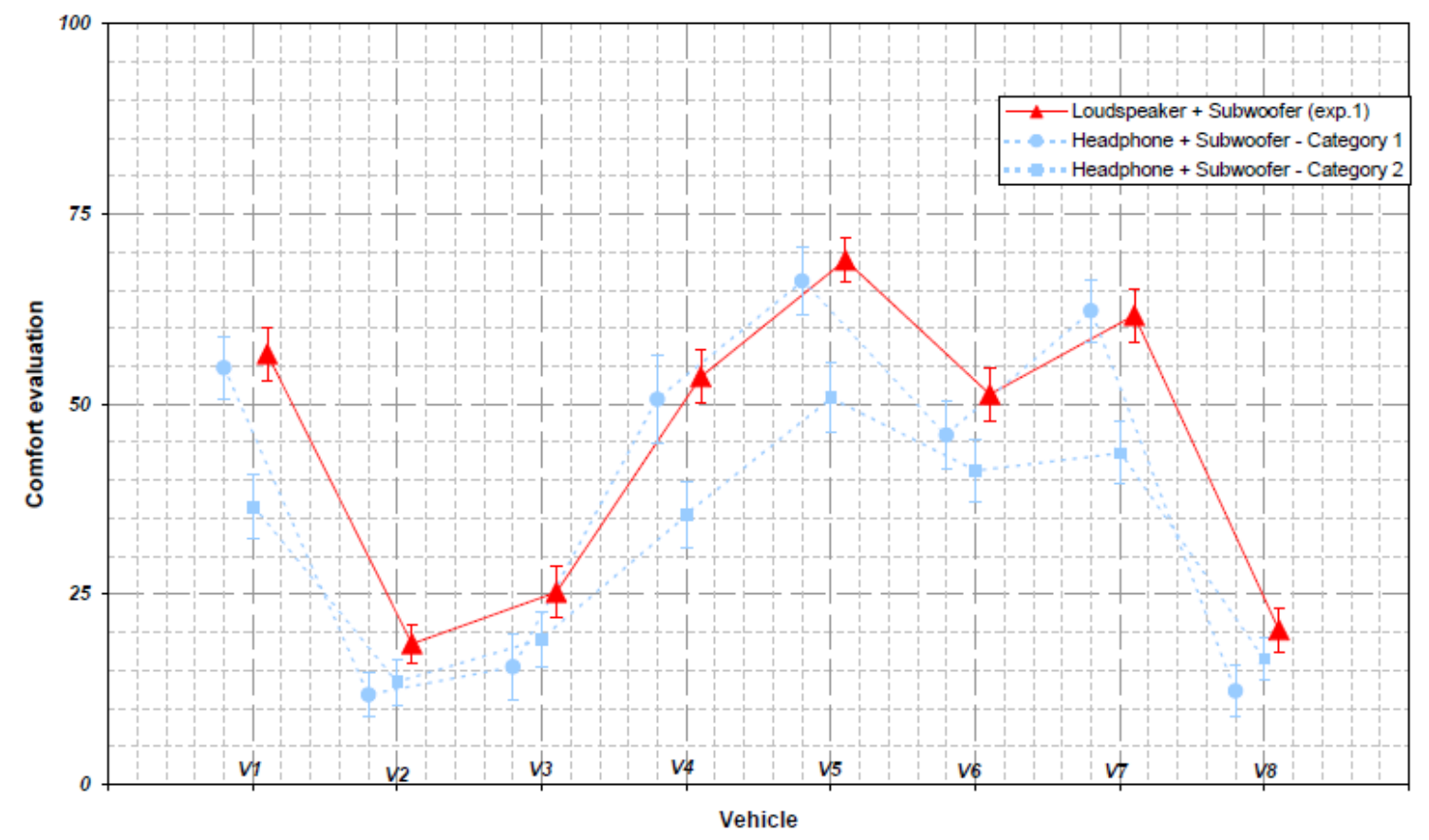

Figure 7: Effect of the sound system 
The degree of discrimination between vehicles was then estimated using average comparison tests (tests HSD - p < 0.05). These revealed a very large degree of discrimination in each test (between five and eight groups across all of the tests). This finding was valid for both participant categories. These were then treated together for the rest of the analysis. The comparison between the degree of discrimination of vehicles from one experiment to the other suggested that the type of sound system has no bearing on this parameter. Therefore, in terms of results, either sound system may be used.

Before gauging the effect of the various visual scenes with precision it is necessary to establish an overview of each of the parameters relating to the presentation of the stimuli. Analyses of variance were calculated on the data for each test. These ANOVA consisted of a comparison of the statistical gaps with regard to the 60 participants (factor taken as random), eight vehicles and two repetitions. The main effects of the factors, along with the order 1 interactions, were included in the model.

The ANOVA results were identical across the three tests. The Vehicle effect was systematically significant, while the Repetition factor was not. The "Vehicle" factor again accounted for the vast majority of the result dispersion. Finally, appropriate additional analyses found that the effects of repetitions need not be taken into account.

\subsubsection{TyPE OF VISUAL SCENE}

Internal mapping was performed for the results obtained for each road. In all cases the first axis of the arrival space represented around half of the inertia of the ratings (Road A and C: $60 \%$, Road D: $55 \%)$. Axis 2 accounts for about $20 \%(\operatorname{Road}$ A: $16 \%$, Road C: $15 \%$ and Road D: $21 \%$ ). This slight reduction of inertia from axis 1 for Road D is not significant. Lastly, the average comparison tests (HSD - P $<0.05$ ) found that the degree of discrimination for vehicles was identical across the three tests (6 groups). In conclusion, the different combinations of visual contexts did not have a significant influence on participants' consensus or vehicle discrimination.

Figure 8 shows the comfort ratings for each vehicle for Roads A, C and D. This parameter exerted little influence compared to the vehicle one. However, for all vehicles the comfort ratings for Road C were slightly lower than for Road A and D. Moreover, the D curve was slightly higher than the other two graphs. Average comparison tests (HSD - P < 0.05) showed that none of these differences between Roads A and C were significant. This was also the case for between Roads A and D (except V1). Finally, there was also no difference between Roads $\mathrm{C}$ and D for half of vehicles (V2, V4, V5 and V7). Therefore, it can be seen that the observed variances were not sufficient for them to be significant. 


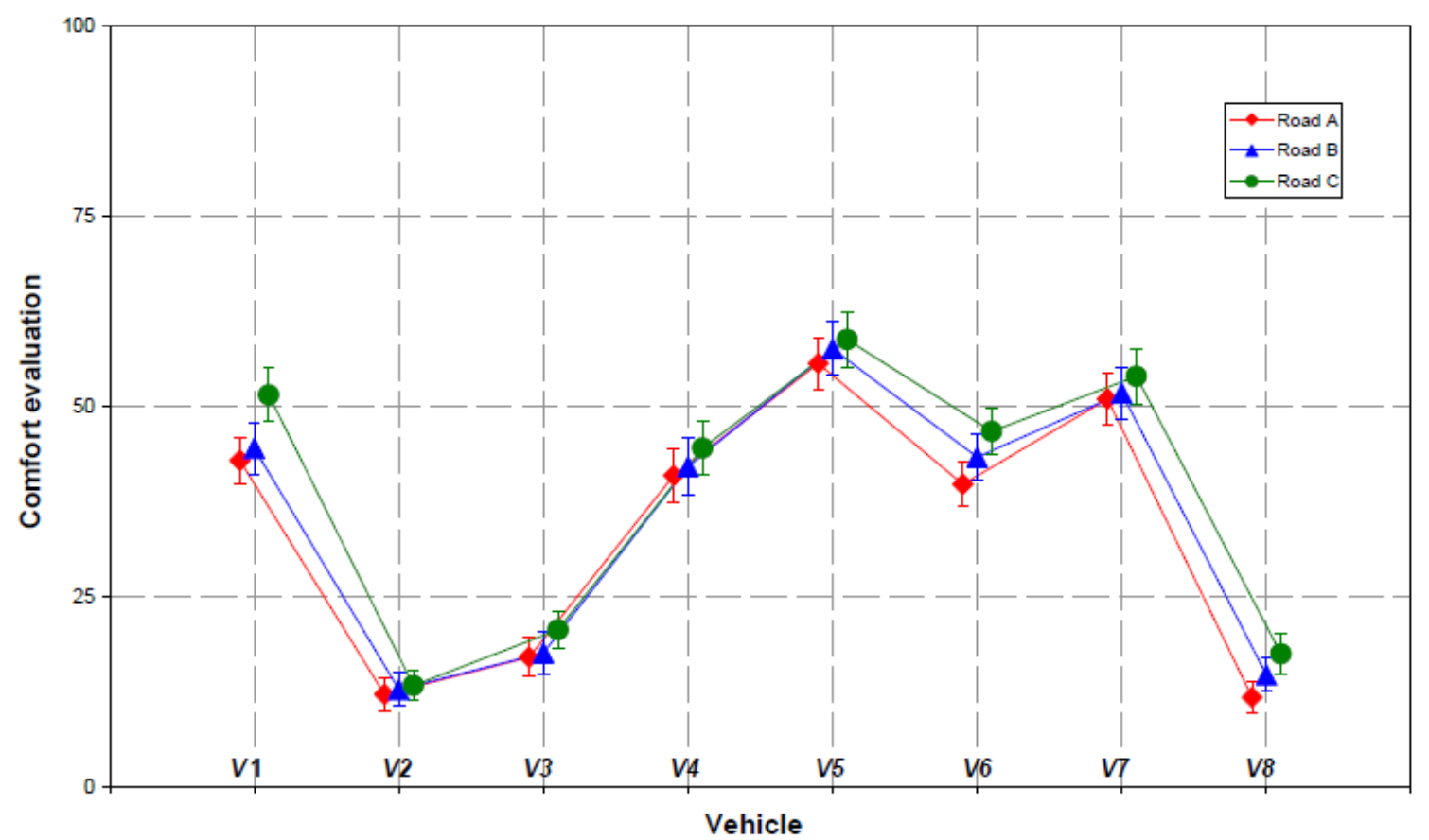

Figure 8: Effect of the type of visual scene

\section{Discussion}

\subsection{EXPERIMENT 1}

The first experiment found a high degree of consensus and vehicle discrimination among tested participants. Regardless of the mode presented, the level of vibration emerged as the crucial factor in assessing overall comfort. These results are comparable to those attained by Genuit et al. in similar condition [1].

Combining the visual mode with the vibration and acoustic modes only leads to a small bearing on rating and a slight increase in vehicle discrimination. These effects are lower than those reported by Ellemeier et al. and Menzel et al., who used visual displays with different semantic content during sound assessments [3, 4]. These results are not necessarily contradictory if we bear in mind that the same video was used with all sound and vibration stimuli in this experiment.

Interactions between sound and vibration modes are also highlighted. Their influences are low and no trend was discernible. The acoustic mode was very significant for only one of the vehicles but no relevant physical indicator could be identified to explain this phenomenon.

No interaction model could be arrived at, owing to the uneven spread of the stimuli levels. However, the results were in line with literature evidence on this participant. Various people have observed significant contributions of vibration and acoustic levels through analyses of artificial or real signals $[15,16,17,18,19]$. In spite of the extensive variety of stimuli used, the models (linear and more complex) make it possible to predict the contribution of sound and vibrations to comfort or nuisance evaluations. While these models systematically harness both vibration and sound levels, the authors sometimes reports that one or other of these modes has a greater bearing. The domination of the vibration mode that is sometimes observed seemed to be amplified here by the weakness of the sound levels used. With 
equivalent vibration levels, Howarth et al., [16], as an example, use sound stimuli of levels up to $79 \mathrm{~dB}(\mathrm{~A})$ while the highest sound level used in this experiment did not exceed $57 \mathrm{~dB}(\mathrm{~A})$.

\subsection{EXPERIMENT 2}

The second experiment demonstrated that combining different visual settings with vibroacoustic stimuli, no matter how much they were opposed, only exerted a small influence on participants' comfort. The addition of a new visual setting only engenders a small rise or fall in overall comfort, which is only significant in a minority of vehicles.

While the effects of visual settings do accurately reflect a participant's expectations with regard to the ride conditions presented to him or her (as in the assumption put forward), it is likely that presenting a situation that is on the face of its comfortable leads to harsher ratings being awarded. Conversely, using a visual display of a heavily deteriorated road evokes greater tolerance and translates into slightly higher comfort ratings. Such effects match increases and drops in the disturbance reported in such situations (sounds are associated with images representing very different degrees of urbanisation) [2]. However, although the situations tested in our experiment were artificially exaggerated with regard to reality, the expressions of the effects were still very low and were only significant in a minority of vehicles.

Finally, with regard to the loudspeakers, using headphones considerably increased variations from one individual to another. No similar effect has been reported in literature. Beyond that, the sound system had no significant impact on assessments.

\section{Conclusion}

These two experiments enabled us to appreciate the respective roles of sound, vibration and visual modes on assessments of vibro-acoustic comfort in simulators. With regard both to immersion and contextualisation, the effects of the visual mode are so low-scale as to be insignificant. Accordingly, they may be ignored in future similar experiments. Sound may be conveyed through a headphones or a transauralized loudspeaker system.

Going forward, work will focus on the relative contributions of vibrations and sounds to automobile driving comfort. Vibration level was shown to be paramount, however it was shown that there was strong interactions between it and the sound mode. It is now important to build a model of these interactions in order to establish criteria for automobile vibroacoustic comfort.

\section{AKNOWLEDGEMENTS}

This study was conducted while the first author was a Ph.D. student in PSA Peugeot-Citroën. His Ph. D. was partly founded by the French National Agency for Research and Technology. 


\section{REFERENCES}

[1] K. Genuit, A. Fiebig, The influence of combined environmental stimuli on the evaluation of acoustical comfort: case studies carried out in an interactive simulation environment. Int. J. Véhicle Noise and Vibration 3 (2007), 119-129.

[2] S. Viollon, C. Lavandier, Carolyn Drake, Influence of visual setting on sound ratings in an urban environment. Applied acoustics, 63 (2002), 493-511.

[3] W. Ellermeier, S. V. Legarth, Visual biais in subjective assesments of automotive sounds. Proceedings of Euronoise, Tampere, June 2006.

[4] D. Menzel, H. Fastl, R. Graf, J. Hellbrück, Influence of vehicle color on loudness judgments. J. Acoust. Soc. Am. 123 (5), 2008, 2477-2479.

[5] C. Patsouras, T. Filippou, H. Fastl, Influences of color on the loudness judgement. Proceedings of $3^{\text {rd }}$ Forum Acusticum, Sevilla, June 2002.

[6] T. Rader, M. Morinaga, T. Matsiu, H. Fastl, S. Kuwano, S. Namba. Crosscultural effects in audio-visual interactions. Proceedings of the meeting of the technical committee on noise and vibration of the acoustical Society of Japan, Tokyo, 2004.

[7] E. Parizet, V. Koehl Influence of train colour on Loudness Judgments. Acta Acustica united with Acustica 97 (2011) 347-349.

[8] D. Warren, T. Mc Carthy, R. Welch, Discrepancy and nondiscrepancy methods of assessing visual-auditory interaction. Perception and Psychophysics 33 (1983) 413-419.

[9] E. Parizet, J. Brocard, B. Piquet, Influence of noise and vibration to comfort in diesel engine cars running at idle. Acta Acustica united with Acustica 90 (2004) 987-993.

[10] H. Möller, Fundamentals of binaural technology. Applied Acoustics 36 (1992) 171-218.

[11] International Organization for Standardization ISO 532, Methods for calculating loudness level, 1975.

[12] International Organization for Standardization ISO 2631-1, Mechanical vibration and shock - evaluation of human exposure to whole-body vibration - part 1: general requirements, 1997.

[13] M. Griffin, Handbook of human vibration. Academic Press, 1996.

[14] Fields J.M., De Jong R.G., Gjestland T., Flindell I.H., Job R.F.S., Kurra S., Lercher P., Vallet M., Yano T., Guski R., Felscher-Suhr U., Schumer R., Standardized generalpurpose noise reaction questions for community noise surveys : research and a recommendation, J. Sound and Vibration 242 (2001), 641-679.

[15] T. Dempsey, J. Leatherwood, S. Clevenson, Development of noise and vibration ride confort criteria. J. Acoust. Soc. Am. 65 (1979) 124-132.

[16] H. Howarth, M. Griffin, Subjective response to combined noise and vibration: summation and interaction effects. Journal of Sound and Vibration 143 (1990) 443-454.

[17] H.Howarth, M.. Griffin, The relative importance of noise and vibration from railways. Applied Ergonomics 21 (1990) 129-134.

[18] R. Paulsen, J. Kastka, Effects of combined noise and vibration on annoyance. Journal of Sound and Vibration 181 (1995) 295-314. 
[19] J. Leatherwood, S. Clevenson, D. Stephens, The development of interior noise and vibration criteria. NASA Technical Memorandum $\mathrm{n}^{\circ} 102736,1990$ (http://ntrs.nasa.gov/archive/nasa/casi.ntrs.nasa.gov/19910001389_1991001389.pdf). 